\title{
Global Pattern Analysis and Classification of Dermoscopic Images Using Textons
}

\author{
Maryam Sadeghi ${ }^{a, b, c}$, Tim. K. Lee ${ }^{a, b, c}$, David McLean ${ }^{b, c}$, Harvey Lui ${ }^{b, c}$, M. Stella Atkins ${ }^{a}$ \\ ${ }^{a}$ Simon Fraser University, 8888 University Drive, Burnaby, BC, Canada, V5A1S6; \\ ${ }^{b}$ Cancer Control Research, BC Cancer Agency, 675 W. 10th Ave., Vancouver, Canada V5Z1L3 \\ ${ }^{c}$ Photomedicine Institute, Department of Dermatology and Skin Science, UBC, Vancouver, \\ Canada
}

\begin{abstract}
Detecting and classifying global dermoscopic patterns are crucial steps for detecting melanocytic lesions from non-melanocytic ones. An important stage of melanoma diagnosis uses pattern analysis methods such as 7-point check list, Menzies method etc. In this paper, we present a novel approach to investigate texture analysis and classification of 5 classes of global lesion patterns (reticular, globular, cobblestone, homogeneous, and parallel pattern) in dermoscopic images. Our statistical approach models the texture by the joint probability distribution of filter responses using a comprehensive set of the state of the art filter banks. This distribution is represented by the frequency histogram of filter response cluster centers called textons. We have also examined other two methods: Joint Distribution of Intensities (JDI) and Convolutional Restricted Boltzmann Machine (CRBM) to learn the pattern specific features to be used for textons. The classification performance is compared over the Leung and Malik filters (LM), Root Filter Set (RFS), Maximum Response Filters (MR8), Schmid, Laws and our proposed filter set as well as CRBM and JDI. We analyzed 375 images of the 5 classes of the patterns. Our experiments show that the joint distribution of color (JDC) in the $\mathrm{L}^{*} \mathrm{a}^{*} \mathrm{~b}^{*}$ color space outperforms the other color spaces with a correct classification rate of $86.8 \%$.
\end{abstract}

\section{DESCRIPTION OF PURPOSE}

In the last two decades, a rising incidence of malignant melanoma has been observed. Because of the lack of adequate therapies for metastatic melanoma, the best treatment is still early diagnosis and prompt surgical excision of the primary cancer. Dermoscopy (also known as epiluminescence microscopy) is an in-vivo method that has been reported to be a useful tool for the early recognition of malignant melanoma. As diagnostic accuracy with dermoscopy has been shown to depend on the training and the experience of the dermatologist, computer assisted diagnosis (CAD) systems will help less-experienced and un-trained dermatologists. This paper follows the new trend in dermatology: to look for specific patterns in the lesions which will lead dermatologists to an assessment. They take into account the overall general appearance or global patterns of the lesion in order to diagnose the malignancy. ${ }^{1}$ The five global patterns of skin lesions are: ${ }^{2}$

- Reticular pattern or pigment network pattern This is the most common global feature in melanocytic lesions. It is characterized by a pigment network covering most parts of a given lesion. Basically, the pigment network appears as a grid of thin brown lines over a diffuse light brown background.

- Globular pattern The globular pattern is characterized by the presence of numerous, variously sized, round to oval structures with various shades of brown and gray-black coloration.

- Cobblestone pattern This is quite similar to the globular one but is composed of closely aggregated, larger, somewhat angulated globules resembling a cobblestone.

- Homogeneous pattern this pattern appears as diffuse pigmentation, which might be brown, grey-blue, grey-black ,or reddish black pigmentation in the absence of pigment network or other distinctive local features.

- Parallel pattern It is found on the palms and soles due to the particular anatomy of these areas.

Medical Imaging 2012: Image Processing, edited by David R. Haynor, Sébastien Ourselin,

Proc. of SPIE Vol. 8314, 83144X - (C) 2011 SPIE · CCC code: 0277-786X/11/\$18 - doi: 10.1117/12.911818

Proc. of SPIE Vol. $831483144 \mathrm{X}-1$ 


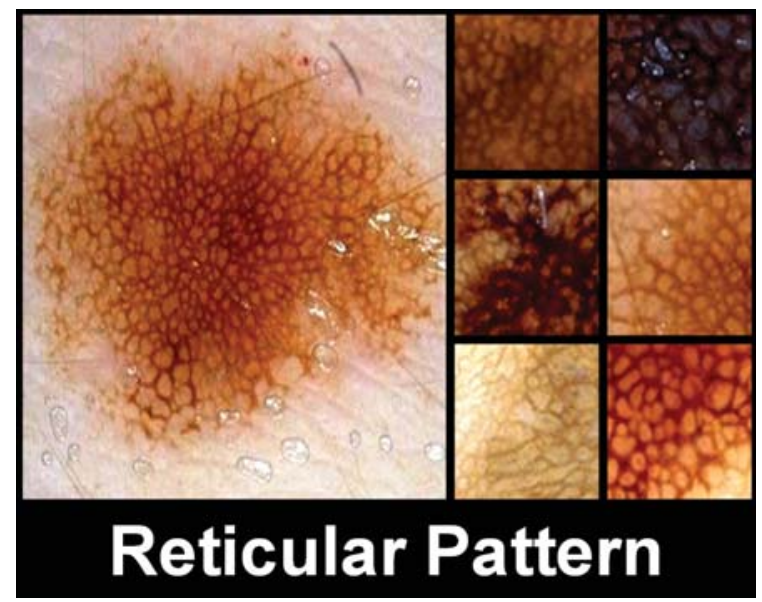

(a)

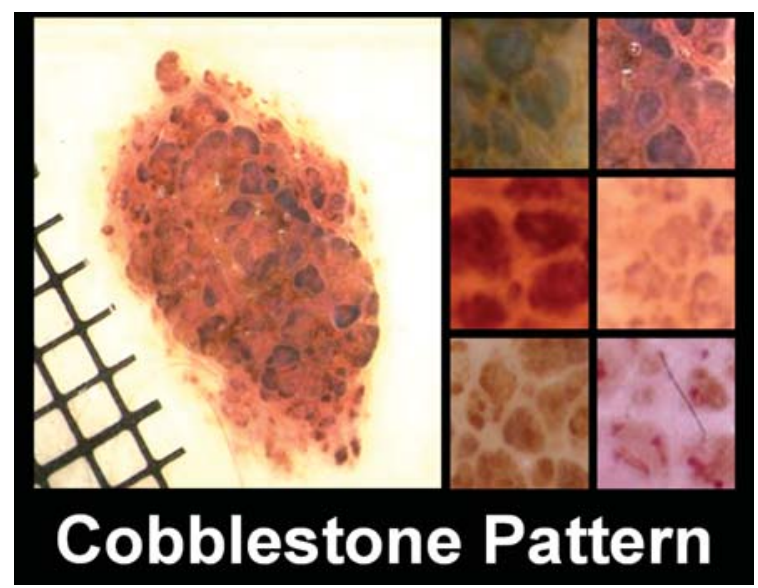

(c)

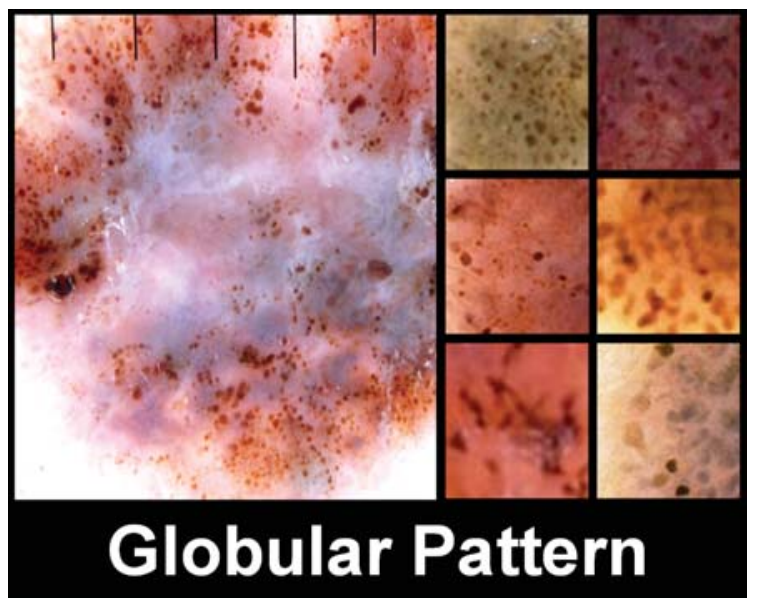

(b)

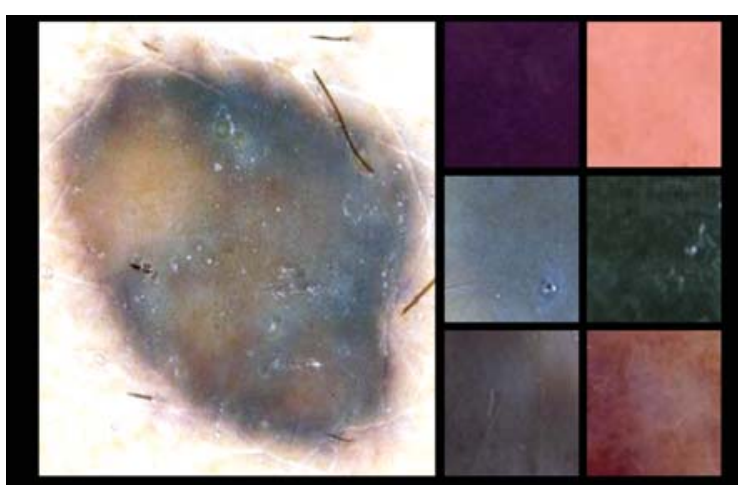

\section{Homogeneous Pattern}

(d)

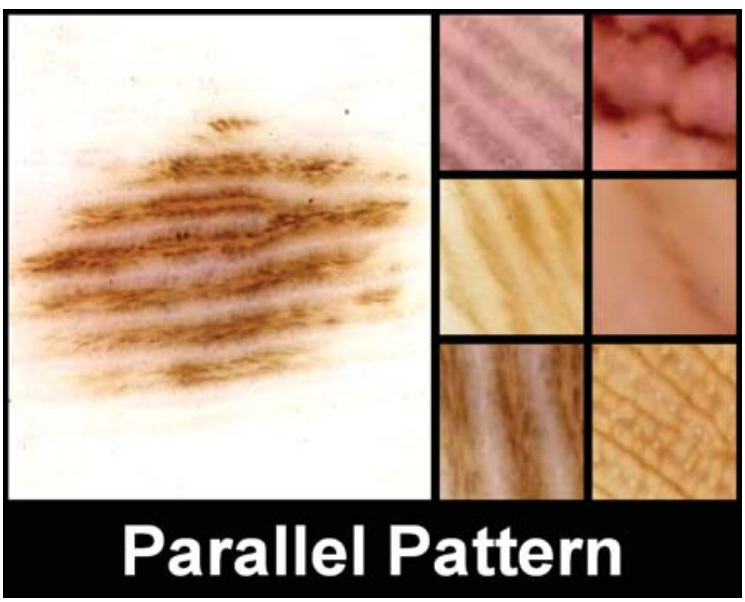

(e)

Figure 1. Skin lesions presenting each one of the 5 global patterns:(a) reticular, (b) globular, (c) cobblestone, (d) homogeneous, and (e) parallel. 


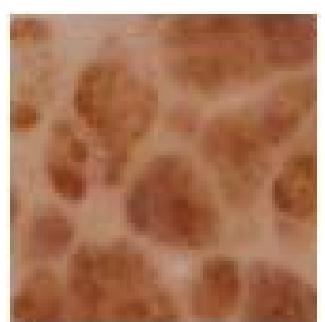

(a)

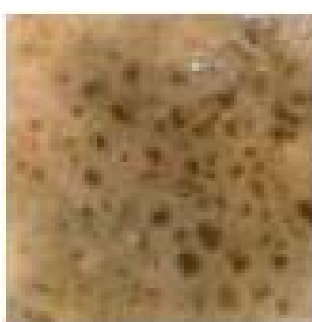

(b)

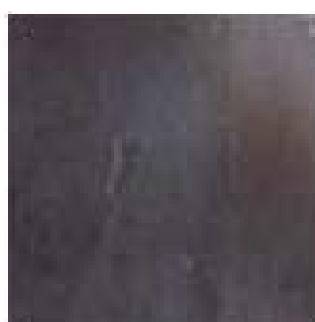

(c)

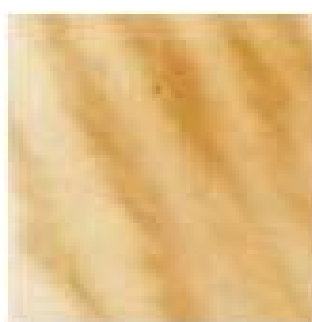

(d)

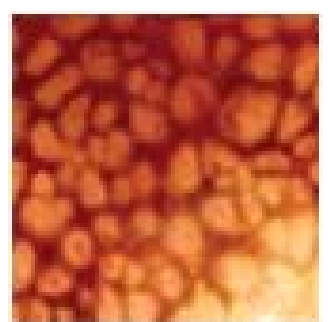

(e)

Figure 2. Dermoscopic $81 * 81$ image samples of each type of patterns to classify: (a) cobblestone pattern, (b) globular pattern, (c) homogeneous pattern, (d) parallel pattern and (e) reticular pattern.

An illustration of these patterns is presented in Fig. 1. The focus of this paper is on automated detection of these 5 patterns using a novel combination of texture features. Other patterns of skin lesions such as starburst, are not included in the experiments reported here.

\subsection{Previous work}

To the best of our knowledge, there is only one reference in the literature that addresses the problem of lesion texture classification into the 5 classes of the global patterns: reticular, globular, cobblestone, homogeneous, and parallel. ${ }^{3}$ Serrano et. al extended Markov random field (MRF) to classify dermoscopic patterns of color skin images. First, each image plane in $\mathrm{L}^{*} \mathrm{a}^{*} \mathrm{~b}^{*}$ colour space is modeled as a MRF following a finite symmetric conditional model (FSCM). Coupling of colour components is taken into account by supposing that features of the MRF in the three colour planes follow a multivariate normal distribution. The best classification rate is $86 \%$ on average over 100 tiles (sized 40x40). However it is not clear how the tiles were selected. It could be the tiles were difficult, real-world examples, or that they were textbook-like definitive exemplars. If tiles are chosen from only one image, it is not as challenging as choosing tiles from different lesions. Also their proposed method is not scale invariant.

\section{METHODS}

In order to classify the five global patterns of dermoscopic images, a statistical approach based on texton classification is followed where texture features are modeled by the joint probability distribution of filter responses. This distribution is represented by texton (cluster center) frequencies, and textons and texture models are learnt from training images. The classification of an unseen image proceeds by mapping the image to a texton distribution and comparing this distribution to the learnt models. In this paper, a pattern recognition algorithm to detect different colour textured patterns, is verified using a set of different filter banks to create the texton dictionary.

To this purpose, a texton-based classification in the $\mathrm{L}^{*} \mathrm{a} * \mathrm{~b}^{*}$ colour space and the gray image using different filter banks is performed and results are compared. The classification method is divided into a learning stage and a classification stage. First, a set of 81 pixels x 81 pixels images representing the 5 patterns are assembled. In the learning stage, training images are convolved with a filter bank to generate filter responses. Exemplar filter responses are chosen as textons (via K-Means clustering) and are used to label each filter response, and thereby every pixel, in the training images. The histogram of texton frequencies is then used to form models corresponding to the training images. In the classification stage, the same procedure as in the training stage is followed to build the histogram corresponding to the unseen image. This histogram is then compared with the models of the texton dictionary. A nearest neighbor classifier is used and the Chi-square ( $\left.\chi^{2}\right)$ statistic is employed to measure distances. Figure 3-a shows the leraning step of texton models and 3-b illustrates the last step of the texton algorithm where the texton profile of an unseen image matches of the best model of a reticular texton which is circled in green. We compared different choices of filter banks and verified them using a database of real dermoscopic images in the following sections. 


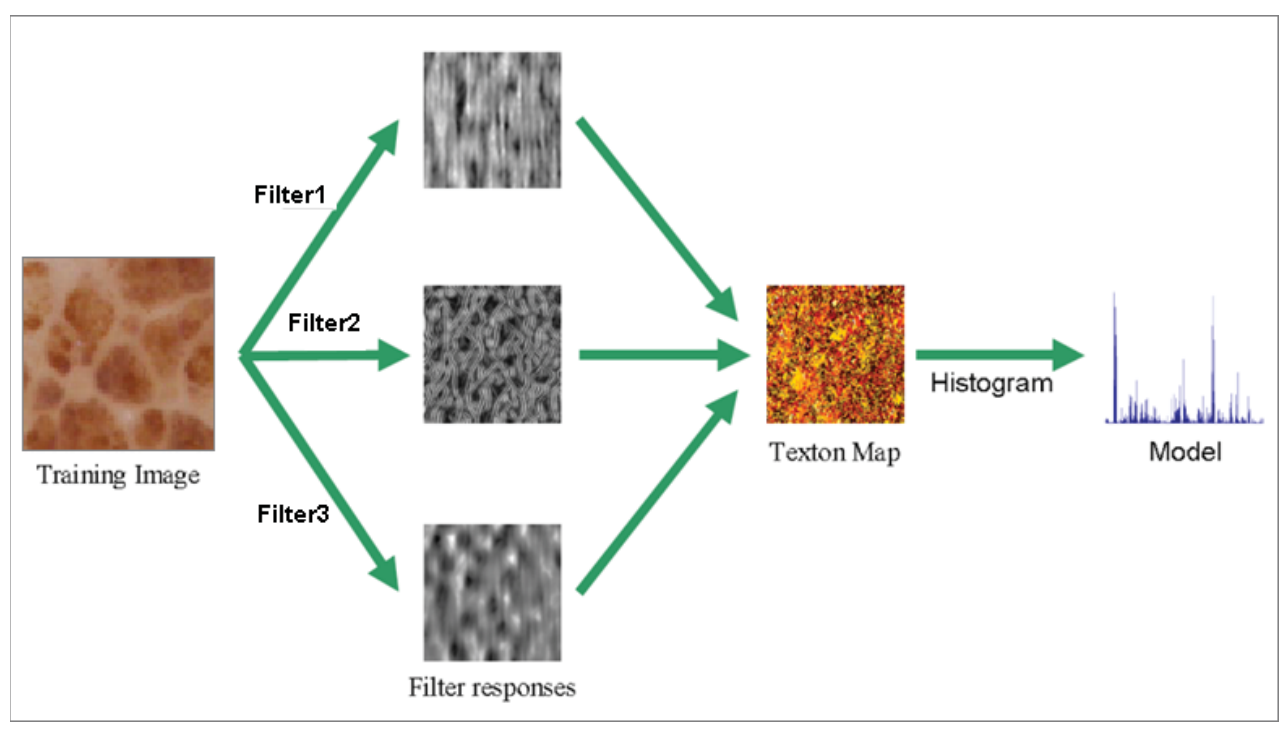

(a)

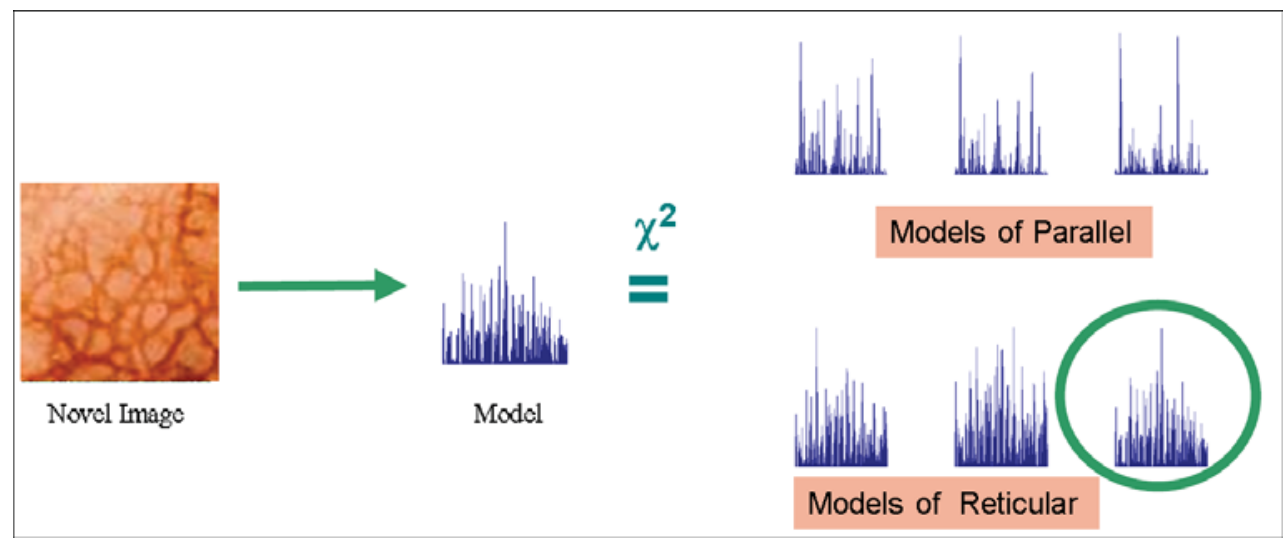

(b)

Figure 3. (a) shows the training step (learning models) and (b) illustrates the test step of the texton algorithm.

\subsection{Filter banks}

The seven filter sets that will be compared are: those of Leung and Malik (LM) ${ }^{4}$ Root Filter Set (RFS), and Maximum Response filters (MR8), ${ }^{5}$ and our proposed filter set; all of which are not rotationally invariant. We also compare rotationally invariant filters proposed by Laws ${ }^{6}$ and $\mathrm{Schmid}^{7}$ as well as texture specific filters learnt by Convolutional Restricted Boltzmann Machine $(\mathrm{CRBM})^{8}$. Our filter bank will be described in this summary, while other filter sets will be described in details in the full paper. All the filter sets will be assessed by their classification performance using textons clustered in their response spaces. In this paper, we constructed a filter bank of $18+18+3$ filters (L1 normalized) to detect average intensity, edges, spots, wave, meshes and ripples of dermoscopy structures. The three different types of filters used in our filter banks are shown in Figure 4. (1) Oriented odd-symmetric filters are at 3 scales and 6 orientations, modeled as rotated copies of the horizontal filter (Gaussian multiplied by its first derivative). A ratio of 3 for $\sigma_{2}: 3 * \sigma_{1}$ was used. We set the 3 scales to be a "half-octave" apart. (2) Oriented even-symmetric filters are at 3 scales and 6 orientations, again rotated copies of a horizontal filter(Gaussian multiplied by its second derivative). (3) Radially symmetric center-surround filters at 3 scales, each modeled as a "Difference of Gaussians" (DOG).

We have also used another method that gives object specific features, (CRBM) created by Norouzi et. al. ${ }^{8}$ 


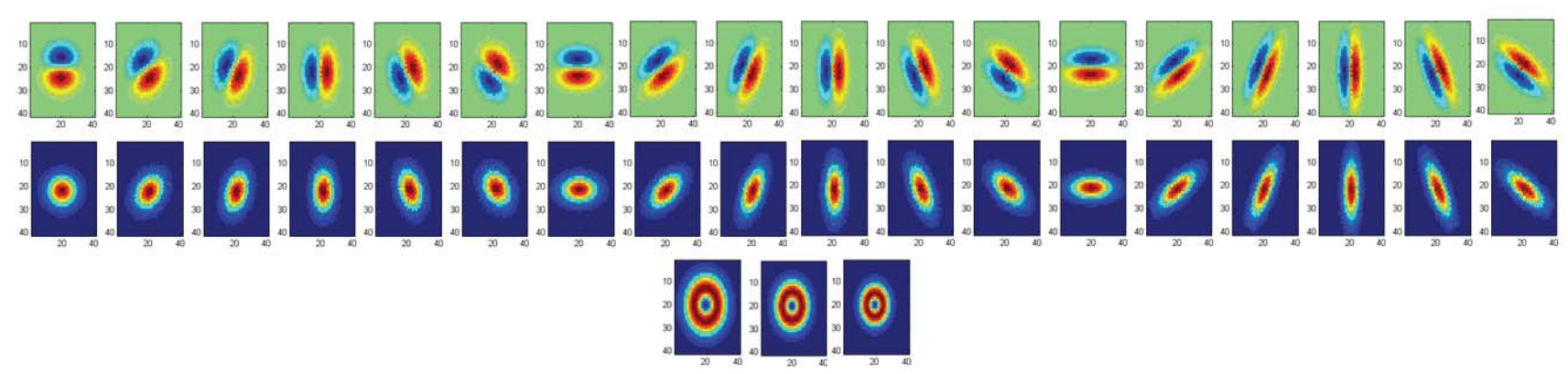

Figure 4. Demonstrates our customized filter bank.

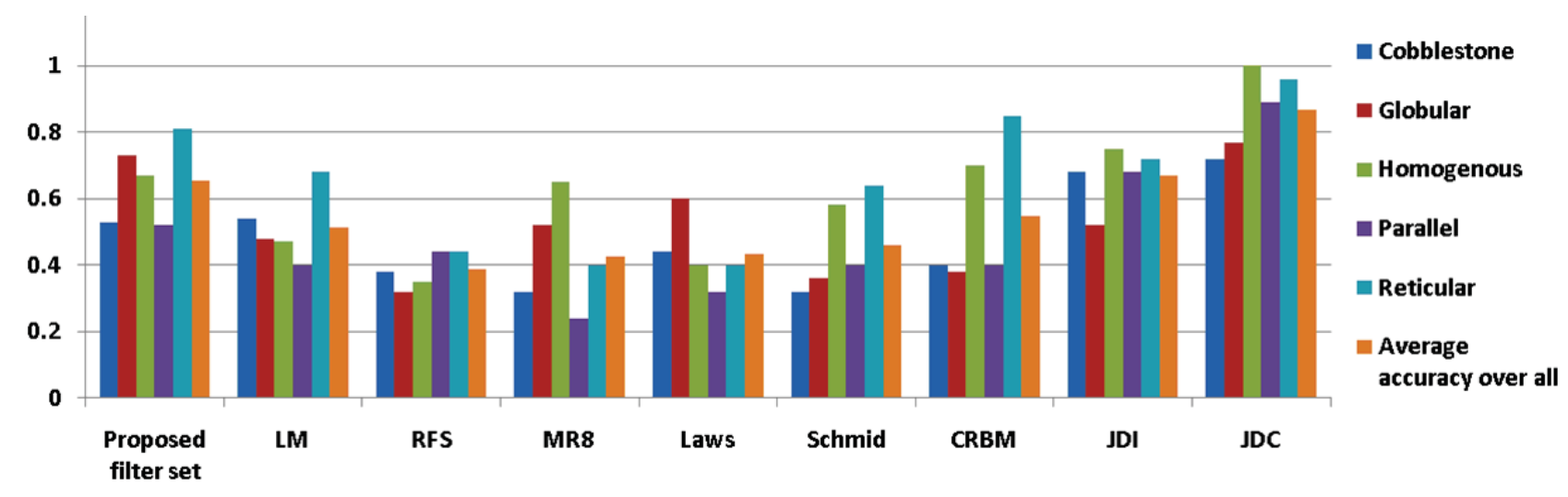

Figure 5. Results of the different methods of the experiment. Each column represents one of the 5 patterns and y-axis shows the accuracy of the clasification methods.

This method learns features specific to an object class. An extension to Restricted Boltzmann Machine model is presented by introducing weight sharing to define features that are replicated over spatial neighborhoods. A set of filters are learned which are tuned to represent a particular object class. We have used these object specific filters to create textons and classify the dermoscopic images.

We also created the texton directory by extending Varma et al.'s extremely compact, intensity filter bands $(\text { JDI })^{9}$ (starting from $3 \times 3$ pixels) to 2 D feature vectors with color components (JDC). Verma et al demonstrated that the joint distribution of intensity values outperformed classical filter banks with large support.

\section{RESULTS}

We verified and compared the above mentioned filter banks on a database containing 375 patches of $81 * 81$ pixels from 325 dermoscopic images sampling the following patterns: reticular, globular, cobblestone, homogeneous and parallel. 75 patches of each reticular, globular, homogeneous and parallel were chosen randomly from 75 different images without any overlap and the 75 patches of the cobblestone pattern is chosen from 25 images.

The data set used in this experiment has a high variance in the appearance and scale. Also, there is a high intra-class variation and inter-class similarities that make the classification task hard for textures of the lesion images. Dots and globules are common structures of skin lesions which are present in most of the images of the data set. Figure 5 shows results of the correct classification rate for the algorithm with our proposed filter bank, LM, RFS, MR8, Laws, Schmid, CRBM, JDI, and JDC. We used 3-fold cross-validation to evaluate the texton method. The algorithm obtained satisfactory results with JDC in $\mathrm{L}^{*} \mathrm{a}^{*} \mathrm{~b}^{*}$ space, with success rate of $86.8 \%$. With a different setting of 75 images $31^{*} 31$ pixels ( 15 patches of each pattern from the same image) the average accuracy is $93.1 \%$. 


\section{CONCLUSION}

We evaluated texton-based classification to detect 5 global (reticular, globular, cobblestone, homogeneous, and parallel) patterns of dermoscopic images. In particular, we tested a set of 9 state of the art filter sets, including two of our proposed sets, on a set of 375 texture patches obtained from 325 challenging dermoscopic images. Our experiment showed that our second proposal set, based on texton directory, achieved the best performance, an average accuracy of $86.8 \%$ in a 3 -fold cross-validation. This method can be used as a part of automatic diagnosis system for classifying moles and skin cancer detection.

\section{ACKNOWLEDGEMENTS}

This work was funded by the Canadian Natural Sciences and Engineering Council (NSERC), CIHR-Skin Research Training Centre (SRTC) and a grant from the Canadian Health Research Project (CHRP).

\section{REFERENCES}

1. R. Braun, H. Rabinovitz, and etal, "Dermoscopy of pigmented skin lesions," J Am Acad Dermatol 52(1), pp. 109-121, 2005.

2. G. Argenziano, H. Soyer, et al., "Interactive atlas of dermoscopy," EDRA-Medical Publishing and New Media, Milan, 2000.

3. C. Serrano and B. Acha, "Pattern analysis of dermoscopic images based on markov random fields," Pattern Recognition 42(6), pp. 1052-1057, 2009.

4. T. Leung and n. J. Malik "Representing and recognizing the visual appearance of materials using threedimensional textons," nternational Journal of Computer Vision 43(1), pp. 29-44, 2001.

5. M. Varma and A. Zisserman, "A statistical approach to texture classification from single images," International Journal of Computer Vision: Special Issue on Texture Analysis and Synthesis 62, pp. 61-81, April 2005.

6. M. Anantha, R. Moss, and W. Stoecker, "Detection of pigment network in dermatoscopy images using texture analysis," Computerized Medical Imaging and Graphics 28(5), pp. 225-234, 2004.

7. P. Schmid, "Segmentation of digitized dermatoscopic images by two dimensional color clustering," Computerized medical imaging and graphics (171), p. 18:164, 1999.

8. M. Norouzi, M. Ranjbar, and G. Mori, "Stacks of convolutional restricted boltzmann machines for shiftinvariant feature learning," in Computer Vision and Pattern Recognition, 2009. CVPR 2009. IEEE Conference on, pp. 2735-2742, IEEE, 2009.

9. M. Varma and A. Zisserman, "Texture classification: Are filter banks necessary?," IEEE Conference on Computer Vision and Pattern Recognition 2, pp. 691-698, June 2003. 